Research article

Open Access

\title{
Knee meniscal extrusion in a largely non-osteoarthritic cohort: association with greater loss of cartilage volume
}

\author{
Changhai Ding ${ }^{1}$, Johanne Martel-Pelletier², Jean-Pierre Pelletier², François Abram³ ${ }^{3}$ Jean- \\ Pierre Raynauld ${ }^{2}$, Flavia Cicuttini ${ }^{4}$ and Graeme Jones ${ }^{1}$
}

\author{
${ }^{1}$ Menzies Research Institute, University of Tasmania, 199 Macquarie Street, Hobart 7000, Australia \\ 2Osteoarthritis Research Unit, University of Montreal Hospital Centre, Notre-Dame Hospital, 1560 Sherbrooke St East, Montreal H2L 4M1, Canada \\ ${ }^{3}$ ArthroVision Inc., 1871 rue Sherbrooke Est, Montreal H2K 1B6, Canada \\ ${ }^{4}$ Department of Epidemiology and Preventive Medicine, Monash University Medical School, Commercial Road, Melbourne 3181, Australia
}

Corresponding author: Changhai Ding, chding@utas.edu.au

Received: 31 Oct 2006 Revisions requested: 12 Jan 2007 Revisions received: 30 Jan 2007 Accepted: 2 Mar 2007 Published: 2 Mar 2007

Arthritis Research \& Therapy 2007, 9:R21 (doi:10.1186/ar2132)

This article is online at: http://arthritis-research.com/content/9/2/R21

(C) 2007 Ding et al.; licensee BioMed Central Ltd.

This is an open access article distributed under the terms of the Creative Commons Attribution License (http://creativecommons.org/licenses/by/2.0), which permits unrestricted use, distribution, and reproduction in any medium, provided the original work is properly cited.

\begin{abstract}
We conducted a longitudinal study (duration 2 years), including 294 individuals (mean age 45 years, 58\% female), in order to examine associations between meniscal extrusion, knee structure, radiographic changes and risk factors for osteoarthritis (OA) in a largely non-osteoarthritic cohort. Meniscal extrusion, tibiofemoral cartilage defect score and cartilage volume, and tibial plateau bone area were determined using T1-weighted fat-saturated magnetic resonance imaging. At baseline the presence of medial meniscal extrusion was significantly associated with body mass index (odds ratio [OR] per $\mathrm{kg} / \mathrm{m}^{2}=1.13,95 \%$ confidence interval $\left.[\mathrm{Cl}]=1.02-1.25\right)$, past knee injury (positive versus negative history: $\mathrm{OR}=3.73$, $95 \% \mathrm{Cl}=1.16-11.97$ ), medial tibial bone area (OR per $\mathrm{cm}^{2}=$ $1.37,95 \% \mathrm{Cl}=1.02-1.85$ ), and osteophytes (OR per grade $=$ $4.89,95 \% \mathrm{Cl}=1.59-15.02)$. Two-year longitudinal data

revealed that medial meniscal extrusion at baseline was associated with a greater rate of loss of medial tibiofemoral cartilage volume (extrusion versus no extrusion: $-1.4 \% /$ year; $P<$ $0.05)$ and greater risk for increased medial femoral cartilage defects $(\mathrm{OR}=2.59,95 \% \mathrm{Cl}=1.14-5.86)$ and lateral tibial cartilage defects $(\mathrm{OR}=2.64,95 \% \mathrm{Cl}=1.03-6.76)$. However, the latter two associations became nonsignificant after adjustment for tibial bone area and osteophytes. This study suggests that increasing body mass index and bone size, past knee injury, and osteophytes may be causally related to meniscal extrusion. Most importantly, meniscal extrusion at baseline is associated with greater loss of knee cartilage over 2 years, and this seems to be mediated mostly by subchondral bone changes, suggesting extrusion represents one pathway between bone expansion and cartilage loss.
\end{abstract}

\section{Introduction}

Knee osteoarthritis (OA) is a common chronic disease that is characterized by whole-organ abnormalities [1], including cartilage lesion and loss, osteophytes, synovial and subchondral bone alterations, and meniscal tears and extrusion. Meniscal extrusion is where the meniscus is partially or totally displaced away from or uncovers the tibial articular cartilage [2]. It has been suggested that significant meniscal extrusion, as seen on magnetic resonance imaging (MRI), occurs more often in patients with knee OA than in normal control individuals, either because it causes the $\mathrm{OA}$ or because laxity of supporting meniscal structures associated with OA predisposes these patients to meniscal extrusion [2]. Meniscal extrusion has been found to be associated with joint space narrowing [2,3], osteophytosis [4,5], the presence of a chondral lesion [6] and meniscal tear $[5,7,8]$ in cross-sectional studies. Longitudinal studies have confirmed that meniscal extrusion is associated with loss of cartilage volume [7,9] and knee cartilage focal loss [10], as determined by MRI. However, most of these studies were conducted in patients with $\mathrm{OA}$, and there are few reports about the relationship between meniscal extrusion and knee structural change in persons without OA. Furthermore, associations between $O A$ risk factors including age, body mass index (BMI), female sex, knee injury, and genetics and meniscal extrusion are unclear, although a cross-sectional study [2]

$\overline{\mathrm{BMI}}=$ body mass index; $\mathrm{MRI}=$ magnetic resonance imaging; $\mathrm{OA}=$ osteoarthritis. 
suggested that meniscal extrusion did not correlate with age and/or weight.

The aim of the present longitudinal study was to describe associations between baseline meniscal extrusion and knee structure, radiographic changes, and $\mathrm{OA}$ risk factors in a largely non-OA cohort.

\section{Materials and methods Patients}

The study was conducted in Tasmania, Australia, primarily in the capital city of Hobart, from June 2000 until December 2001. Participants were selected from two sources. Approximately half were the adult children of patients who had undergone knee replacement for primary knee OA at any Hobart hospital during the years from 1996 to 2000 (offspring: $n=$ 186 , age 45 years, $59 \%$ female). The parents' diagnosis was confirmed by reference to medical records of the orthopaedic surgeon and the original radiograph where possible. The other participants were control individuals ( $n=186$; age 45 years, $57 \%$ female) selected at random from the electoral roll. Those selected were eligible to participate if they had no parent with either a history of symptomatic knee OA or a knee replacement for OA. Individuals from either group were excluded on the basis of contraindication to MRI (including metal sutures, presence of shrapnel, iron filings in the eye and claustrophobia). This study was approved by the Southern Tasmanian Health and Medical Human Research Ethics Committee, and all participants provided informed written consent.

The characteristics of the participants were reported previously [11]. The follow-up study was conducted about 2 years later (mean 2.3 years).

\section{Anthropometrics}

Weight was measured to the nearest $0.1 \mathrm{~kg}$ (with shoes, socks and bulky clothing removed) using a set of electronic scales (a single unit; Seca Delta, Model 707; Seca, Hamburg, Germany) calibrated using a known weight at the beginning of each clinic session. Height was measured to the nearest 0.1 $\mathrm{cm}$ (with shoes and socks removed) using a stadiometer. Body mass index (BMl; $\left.\mathrm{kg} / \mathrm{m}^{2}\right)$ was calculated.

\section{Past knee injury}

Our definition of past knee injury was documented in the questionnaire as follows: 'Have you had a previous knee injury requiring non-weight-bearing treatment for more than 24 hours or surgery?'

\section{Radiography}

A standing anteroposterior semiflexed view of the right knee (at $15^{\circ}$ flexion) was performed in all participants at baseline and scored individually for osteophytes and joint space narrowing, as described previously [12]. Each of the following four features was scored on a scale from 0 to $3(0=$ normal and $3=$ severe): medial joint space narrowing, lateral joint space narrowing, medial osteophytes (femoral and tibial combined) and lateral osteophytes (femoral and tibial combined).

\section{Magnetic resonance imaging}

Magnetic resonance imaging (MRI) of the right knee was performed as described previously [13-15]. Knees were imaged in the sagittal plane on a $1.5 \mathrm{~T}$ whole-body magnetic resonance unit (Picker International, Cleveland, $\mathrm{OH}, \mathrm{USA}$ ) using a commercial transmit-receive extremity coil. The following image sequence was used: a T1-weighted fat saturation threedimensional gradient recall acquisition in the steady state; flip angle $55^{\circ}$; repetition time $58 \mathrm{~ms}$; echo time $12 \mathrm{~ms}$; field of view $16 \mathrm{~cm}$; 60 partitions; $512 \times 512$ matrix; acquisition time $11 \mathrm{~min} 56 \mathrm{~s}$; and one acquisition. Sagittal images were obtained at a partition thickness of $1.5 \mathrm{~mm}$ and an in-plane resolution of $0.31 \times 0.31 \mathrm{~mm}(512 \times 512$ pixels $)$. The coronal and axial views were then reformatted.

\section{Meniscal extrusion assessment}

The extent of meniscal extrusion (Figure 1) on the medial or lateral edges of the tibial femoral joint space, not including the osteophytes, was evaluated at baseline and at 2 years by two observers for the anterior, body, and posterior horns of the menisci. A score from 0 to 2 was employed, in which $0=$ no extrusion, 1 = partial meniscal extrusion, and $2=$ complete meniscal extrusion with no contact with the joint space. The intra-reader and inter-reader correlation coefficient ranged from 0.85 to 0.92 for the meniscal extrusion, as reported previously $[7,9]$.

Knee cartilage volume, defects and bone area measurement Knee cartilage volume, defects and bone area were evaluated at baseline and 2 years by one observer (CD) who was blinded to the meniscal assessment. Knee cartilage volume was determined by means of image processing on an independent workstation at baseline and follow up. The volumes of individual cartilage plates (medial tibia and femora, and lateral tibia and femora) were isolated from the total volume by manually drawing dis-articulation contours around the cartilage boundaries on a section by section basis. These data were then resampled by means of bilinear and cubic interpolation (area of $312 \times 312 \mu \mathrm{m}$ by $1.5 \mathrm{~mm}$ thickness, continuous sections) for the final three-dimensional rendering. The coefficients of variation were $2.1 \%$ to $2.2 \%$ [13] for tibial cartilage volume measures and were $1.2 \%$ to $2.6 \%$ [16] for femoral cartilage measures. The percentage change in cartilage volume per year was calculated as follows: $(100 \times$ [(cartilage volume at follow up - cartilage volume at baseline)/cartilage volume at baseline]/time between scans in years).

The cartilage defects (scale from 0 to 4) were graded by two observers at medial tibial and femoral, and lateral tibial and femoral sites, as previously described, with excellent reproducibility [17]: grade $0=$ normal cartilage; grade $1=$ focal 
Figure 1

(a)

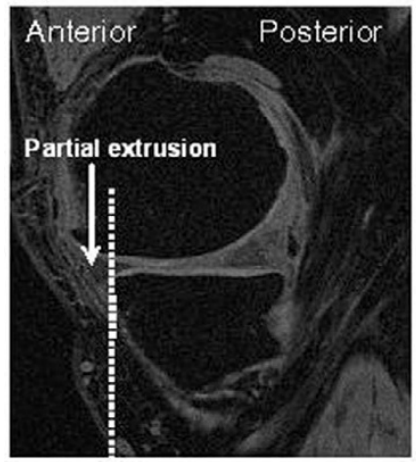

(b)

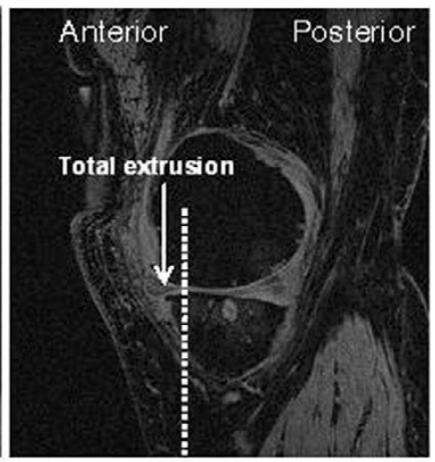

(c)

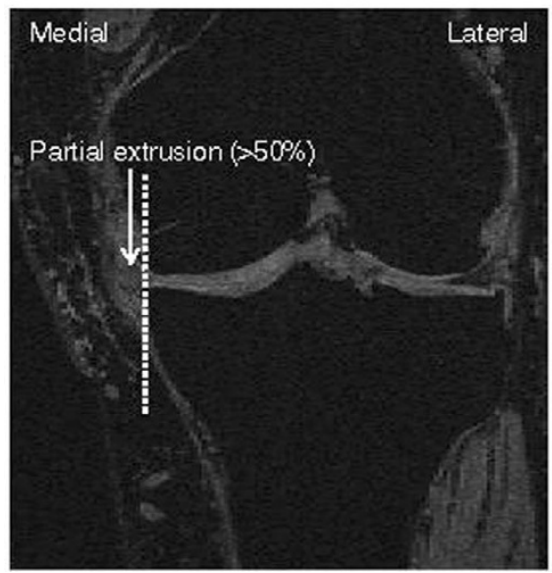

Representations of meniscal extrusion as seen using magnetic resonance imaging. Human knee medial compartment: (a) and (b) sagittal views, and (c) reconstructed coronal view. Partial and total meniscal extrusion at the anterior horn are shown in panels a and b, respectively. Panel c shows a partial (> 50\%) meniscal extrusion at the body site.

blistering and intracartilaginous low-signal intensity area with an intact surface and bottom; grade $2=$ irregularities on the surface or bottom and loss of thickness of less than $50 \%$; grade $3=$ deep ulceration with loss of thickness of more than $50 \%$; and grade $4=$ full-thickness chondral wear with exposure of subchondral bone. Intraobserver reliability (expressed as intraclass correlation coefficient) was 0.89 to 0.94 and interobserver reliability was 0.85 to $0.93[15,17]$. Changes in tibial and/or femoral cartilage defects were calculated by subtracting tibial and/or femoral cartilage defect scores ( 0 to 4 or 0 to 8 scale) at baseline from tibial and/or femoral cartilage defect scores ( 0 to 4 or 0 to 8 scale) at follow up.

The area of medial and lateral tibial plateau bone was measured manually on the three reformatted images closest to tibial cartilage. An average of these three areas was used as an estimate of the tibial plateau bone area $[12,15]$. The coefficients of variation for these measures range from $2.2 \%$ to $2.6 \%$ [13].

\section{Data analysis}

Unpaired $t$-test or $\chi^{2}$ test was utilized for comparison of means or frequencies. Multiple logistic regression analysis was used to examine the associations between the presence or absence of medial meniscal extrusion at baseline and age, sex, family history of OA, past knee injury, BMI, tibial cartilage volume, cartilage defect scores, tibial bone area, radiographic assessment, and change in cartilage defect score before and after adjustment for confounders. Linear regression was utilized to examine the associations between change in cartilage volume and presence of medial meniscal extrusion at baseline before and after adjustment for confounders. A $P$ value less than 0.05 (two-tailed) was considered statistically significant. All statistical analyses were performed on SPSS version 12.0 for Windows (SPSS Inc., Chicago, IL, USA).

\section{Results}

A total of 325 individuals (58\% female) completed the study, with a response rate of $87 \%$. The reasons for loss to follow up (and number of individuals) were as follows: deceased (two); moved interstate (five); claustrophobic (three); and illness (four) and others (no reason). Meniscal extrusion was measured in the first 294 participants (58\% female; offspring $n=$ 135 , control individuals $n=159)$ at both baseline (79\% of all participants) and follow up ( $90 \%$ of all participants). There were no significant differences in demographic or structural factors between the whole cohort and the individuals who were not included (data not shown).

Meniscal extrusion was found at baseline in 23 participants, with 21 (7\% of the total population) at the medial compartment (17 partial at the body site, one partial at body and anterior horn site, and three complete at body site) and two (0.7\%) at the lateral compartment (one partial body site and one complete body site). Medial meniscal extrusion was present in 4\% of individuals without any radiographic changes $(n=242)$ but in $21 \%$ of those with radiographic changes $(n=52 ; P<$ $0.001)$. There was no difference in presence of medical meniscal extrusion between control individuals without a family history of OA (6\%) and offspring with a family history of OA (9\%; $P=0.28)$.

Over 2 years, meniscal extrusion developed in only four participants (1.4\%; all at the body site in the medial compartment); their mean age was 47 years (range 41 to 54 years) and mean BMl was $32 \mathrm{~kg} / \mathrm{m}^{2}$ (range 26 to $45 \mathrm{~kg} / \mathrm{m}^{2}$ ). Three of these four individuals were women; three were offspring of patients with severe knee OA, and one had a past knee injury. Because of the small sample size $(n=4)$, the precise reasons for the occurrence of a new meniscal extrusion could not be determined.

The characteristics of the participants are presented in Table 1. The data show that the participants with and those without medial meniscal extrusion were similar in terms of age, sex, 
Table 1

\begin{tabular}{|c|c|c|c|}
\hline \multirow[t]{2}{*}{ Characteristic } & \multicolumn{2}{|c|}{ Meniscal extrusion at baseline } & \multirow[t]{2}{*}{$P$ value } \\
\hline & Negative $(n=273)$ & Positive $(n=21)$ & \\
\hline Age (years) & $45.0(6.6)$ & $47.2(5.4)$ & 0.148 \\
\hline Sex (female [\%]) & 59 & 43 & $0.150^{*}$ \\
\hline Family history of OA (positive [\%]) & 45 & 57 & $0.284^{*}$ \\
\hline Height $(\mathrm{cm})$ & $169.0(8.5)$ & $172.1(8.2)$ & 0.102 \\
\hline Weight (kg) & $76.6(14.6)$ & $92.7(21.5)$ & $<0.001$ \\
\hline $\operatorname{BMI}\left(\mathrm{kg} / \mathrm{m}^{2}\right)$ & $26.8(4.5)$ & $31.3(7.0)$ & $<0.001$ \\
\hline Knee pain (\%) & 33 & 38 & $0.665^{\star}$ \\
\hline Obese (\%) & 18 & 52 & $<0.001^{*}$ \\
\hline Past knee injury (\%) & 19 & 38 & $0.037^{\star}$ \\
\hline Medial tibial cartilage volume (ml) & $2.2(0.5)$ & $2.4(0.5)$ & 0.051 \\
\hline Lateral tibial cartilage volume (ml) & $2.6(0.7)$ & $2.7(0.6)$ & 0.408 \\
\hline Medial femoral cartilage volume (ml) & $4.6(1.3)$ & $4.7(1.0)$ & 0.632 \\
\hline Lateral femoral cartilage volume (ml) & $4.7(1.3)$ & $5.1(1.0)$ & 0.280 \\
\hline Medial tibial bone area $\left(\mathrm{cm}^{2}\right)$ & $17.3(2.6)$ & $19.8(3.8)$ & $<0.001$ \\
\hline Lateral tibial bone area $\left(\mathrm{cm}^{2}\right)$ & $11.9(2.0)$ & $13.5(2.5)$ & 0.001 \\
\hline Medial tibiofemoral cartilage defect (0-8) & $2.0(0.6)$ & $3.1(1.4)$ & $<0.001$ \\
\hline Lateral tibiofemoral cartilage defect $(0-8)$ & $2.0(0.7)$ & $2.1(0.7)$ & 0.550 \\
\hline Any radiographic osteoarthritis (\%) & 15 & 52 & $<0.001^{*}$ \\
\hline Medial joint space narrowing (\%) & 13 & 38 & $0.001^{*}$ \\
\hline Medial osteophytes (\%) & 4 & 43 & $<0.001^{*}$ \\
\hline Lateral joint space narrowing (\%) & 3 & 5 & $0.737^{\star}$ \\
\hline Lateral osteophytes (\%) & 3 & 14 & $<0.001^{*}$ \\
\hline Change in medial tibial cartilage volume (\%) & $-2.4(4.1)$ & $-4.7(4.4)$ & 0.015 \\
\hline Change in lateral tibial cartilage volume (\%) & $-1.5(3.4)$ & $-1.6(3.8)$ & 0.860 \\
\hline Change in medial femoral cartilage volume (\%) & $-3.3(2.6)$ & $-4.1(3.9)$ & 0.278 \\
\hline Change in lateral femoral cartilage volume (\%) & $-3.3(2.5)$ & $-3.5(3.1)$ & 0.662 \\
\hline Change in medial tibial bone area (\%) & $+0.7(1.8)$ & $+0.2(1.7)$ & 0.258 \\
\hline Change in lateral tibial bone area (\%) & $+0.3(2.8)$ & $+0.5(2.4)$ & 0.781 \\
\hline Change in medial tibiofemoral cartilage defect & $-0.07(0.86)$ & $0.29(1.06)$ & 0.074 \\
\hline Change in lateral tibiofemoral cartilage defect & $-0.02(0.81)$ & $0.50(1.20)$ & 0.007 \\
\hline
\end{tabular}

Values are expressed as mean (standard deviation), except for percentage. ${ }^{\star} \chi^{2}$ test; all others $t$-test. BMl, body mass index; OA, osteoarthritis.

family history of $O A$, height, prevalence of knee pain, baseline cartilage volume, and change in tibial bone area. However, compared with participants with no medial meniscal extrusion, the following parameters were greater in those with meniscal extrusion: BMI, weight, proportion of past knee injury, tibial bone area at baseline, medial tibiofemoral cartilage defect score, prevalence of radiographic OA, medial joint space narrowing and osteophytes at baseline, loss of medial tibial carti- lage volume, and progression of tibiofemoral cartilage defect score.

Age, sex and family history of OA were not significantly associated with baseline medial meniscal extrusion in both univariable and multivariable analyses (Table 2). However, BMI, weight, obesity and our definition of past knee injury were significantly associated with baseline medial meniscal extrusion, 
Table 2

Factors associated with baseline medial meniscus extrusion

\begin{tabular}{|c|c|c|c|}
\hline Factor & Univariable (OR $[95 \% \mathrm{Cl}]$ ) & Multivariable $(\mathrm{OR}[95 \% \mathrm{Cl}])$ & $P$ value \\
\hline Age (per year) & $1.06(0.98-1.13)$ & $1.03(0.94-1.13)$ & 0.579 \\
\hline Sex (female versus male) & $0.52(0.21-1.28)$ & $3.67(0.65-20.80)$ & 0.141 \\
\hline OA family history (+ versus -) & $1.63(0.66-3.99)$ & $0.65(0.21-2.02)$ & 0.452 \\
\hline BMI (per unit) & $1.16(1.07-1.25)^{\star}$ & $1.13(1.02-1.25)^{\star}$ & 0.019 \\
\hline Weight (per kg) & $1.06(1.03-1.09)^{\star}$ & $1.04(1.00-1.08)^{\star b}$ & 0.029 \\
\hline Obese (+ versus -$)$ & $4.90(1.98-12.18)^{\star}$ & $4.87(1.59-14.92)^{\star c}$ & 0.006 \\
\hline Past knee injury (+ versus -) & $2.62(1.03-6.64)^{*}$ & $3.73(1.16-11.97)^{\star}$ & 0.027 \\
\hline Medial tibial cartilage volume (per ml) & $2.06(0.99-4.31)$ & $1.52(0.42-5.54)$ & 0.527 \\
\hline Medial tibial bone area $\left(\right.$ per $\left.\mathrm{cm}^{2}\right)$ & $1.35(1.15-1.59)^{\star}$ & $1.37(1.02-1.85)^{\star}$ & 0.038 \\
\hline Medial radiographic osteoarthritis (+ versus -) & $6.88(2.57-18.40)^{\star}$ & $4.21(1.25-14.21)^{*}$ & 0.021 \\
\hline Medial joint space narrowing (per grade) & $4.27(1.65-11.06)^{\star}$ & $1.97(0.56-6.87)$ & 0.288 \\
\hline Medial osteophytes (per grade) & $9.52(3.49-25.97)^{\star}$ & $4.89(1.59-15.02)^{\star}$ & 0.006 \\
\hline
\end{tabular}

The dependent variable was medial meniscal extrusion. aAjusted by factors listed in the table. ${ }^{b}$ Adjusted for height and factors listed except for body mass index (BMI). cAdjusted for factors listed except for BMI and weight. *Statistically significant associations. Cl, confidence interval; OA, osteoarthritis; OR, odds ratio.

even after adjustment for confounders (Table 2). Specifically, medial meniscal extrusion occurred in $18 \%$ of obese participants $\left(\mathrm{BMI}=30 \mathrm{~kg} / \mathrm{m}^{2} ; n=61\right)$ versus $4 \%$ of nonobese participants (BMI $<30 \mathrm{~kg} / \mathrm{m}^{2} ; n=233$ ), and $13 \%$ of those with past knee injury $(n=60)$ versus $6 \%$ of those without past knee injury $(n=234)$. Moreover, baseline medial meniscal extrusion was significantly associated with medial tibial bone area and medial osteophytes, but was not associated with medial tibial cartilage volume both in univariable and multivariable analyses. It was associated with medial joint space narrowing (19\% versus $5 \%$ medial meniscal extrusion in participants with and without medial joint space narrowing) before (Table 2) and after adjustment for age, sex, family history of OA, BMI, our definition of past knee injury and baseline medial tibial cartilage volume (odds ratio $=3.86 ; P=0.021$ ), but this association became nonsignificant after further adjustment for tibial bone area and osteophytes (Table 2).

Data from Table 3 show that baseline medial meniscal extrusion was significantly associated with baseline medial tibial, femoral and tibiofemoral cartilage defect scores after adjustment for age, sex, family history of OA, BMI, past knee injury and change in cartilage defect scores, and with change in medial femoral and lateral tibial cartilage defect scores after adjustment for age, sex, OA family history, BMI, past knee injury, and baseline cartilage defect scores (Table 3). However, these associations again became nonsignificant after further adjustment for baseline tibial bone area and osteophytes (Table 3).

Prevalent medial meniscal extrusion at baseline was significantly associated with subsequent rate of change in medial femoral and tibiofemoral cartilage volume after adjustment for the confounders (Table 4). In contrast, medial meniscal extrusion at baseline was significantly associated with change in medial tibial cartilage volume in univariable analysis (Table 4 ), but this association became nonsignificant after adjustment for other factors, mostly contributed by BMI and baseline cartilage volume $(\beta=-1.41 \% ; P=0.128)$.

\section{Discussion}

To our knowledge, this is the first time that associations of meniscal extrusion with knee structural changes and risk factors in a largely non-OA cohort have been documented. As may be expected for such individuals, the prevalence of meniscal extrusion was low, predominantly involving partial extrusion. Medial meniscal extrusion was significantly associated with BMI, past knee injury and tibial bone area, as well as with osteophytes and medial tibiofemoral cartilage defect scores. Over 2 years, those with medial meniscal extrusion exhibited greater loss of medial tibiofemoral cartilage volume and greater increase in medial femoral and lateral tibial cartilage defect score than did those without medial meniscal extrusion. The association between meniscal extrusion and knee cartilage defects became nonsignificant after adjustment for bone area and/or osteophytes, suggesting that extrusion may represent one pathway between bone expansion and cartilage loss.

In this largely non-OA cohort, medial and lateral meniscal extrusion occurred in $7 \%$ and $0.7 \%$ of individuals, respectively, and medial meniscal extrusion developed in $1.4 \%$ over 2 years. These prevalence rates are similar to those observed in normal individuals [4] and those with knee pain [18], but far less than those observed in patients with either symptomatic 
Table 3

Associations between baseline medial meniscal extrusion and baseline cartilage defect score and change in cartilage defect score over 2 years

\begin{tabular}{|c|c|c|c|}
\hline Factor & Multivariablea (OR [95\% Cl]) & Multivariableb (OR $[95 \% \mathrm{Cl}]$ ) & Multivariablec (OR [95\% Cl]) \\
\hline \multicolumn{4}{|l|}{ Baseline cartilage defect score } \\
\hline Medial tibial (0-4) & $5.91(2.53-13.77)^{\star}$ & $3.41(1.36-8.57)^{\star}$ & $0.63(0.17-2.32)$ \\
\hline Medial femoral (0-4) & $6.71(2.65-16.99)^{*}$ & $4.62(1.74-12.30)^{\star}$ & $2.39(0.71-8.02)$ \\
\hline Medial tibiofemoral (0-8) & $3.42(1.92-6.10)^{\star}$ & $2.45(1.36-4.40)^{\star}$ & $1.23(0.59-2.53)$ \\
\hline Lateral tibial $(0-4)$ & $1.52(0.59-3.98)$ & $0.97(0.29-3.22)$ & $0.20(0.03-1.37)$ \\
\hline Lateral femoral $(0-4)$ & $1.84(0.78-4.34)$ & $1.30(0.45-3.74)$ & $0.49(0.12-2.05)$ \\
\hline Lateral tibiofemoral (0-8) & $1.33(0.82-2.17)$ & $1.13(0.63-2.05)$ & $0.55(0.22-1.39)$ \\
\hline \multicolumn{4}{|l|}{ Change in cartilage defect score } \\
\hline Medial tibial (0-4) & $1.54(0.57-4.13)$ & $1.24(0.45-3.44)$ & $0.31(0.07-1.42)$ \\
\hline Medial femoral $(0-4)$ & $3.12(1.50-6.49)^{\star}$ & $2.59(1.14-5.86)^{\star}$ & $1.30(0.51-3.35)$ \\
\hline Medial tibiofemoral (0-8) & $1.79(1.06-3.03)^{\star}$ & $1.56(0.88-2.77)$ & $0.85(0.40-1.81)$ \\
\hline Lateral tibial $(0-4)$ & $2.92(1.25-6.79)^{\star}$ & $2.64(1.03-6.76)^{\star}$ & $1.54(0.48-4.95)$ \\
\hline Lateral femoral (0-4) & $2.30(1.10-4.81)^{*}$ & $1.68(0.74-3.79)$ & $1.01(0.39-2.67)$ \\
\hline Lateral tibiofemoral (0-8) & $2.01(1.22-3.31)^{\star}$ & $1.80(1.05-3.08)^{\star}$ & $1.21(0.61-2.42)$ \\
\hline
\end{tabular}

The dependent variable was medial meniscal extrusion. aAdjusted for change in cartilage defect score if associations between baseline cartilage defect scores and baseline meniscal extrusion were determined, or baseline cartilage defect score if associations between changes in cartilage defect score and baseline meniscal extrusion were determined. bFurther adjusted for age, sex, offspring/control status, body mass index and past knee injury. 'Further adjusted for baseline tibial bone area and osteophytes. ${ }^{\star}$ Statistically significant associations. Cl, confidence interval; OR, odds ratio.

or asymptomatic knee OA [2] and in nonadvanced arthritic patients who underwent arthroscopy for other disorders [6]. The difference is most likely due to different disease status and age group in the different cohorts. In the present study, individuals with early radiographic changes had a greater prevalence of medial meniscal extrusion than did those with no radiographic changes.

Any factors that affect meniscal stability and structure may cause meniscal extrusion. In this longitudinal study only four participants developed partial meniscal extrusion over 2 years, so we could not document any predictors of meniscal extrusion. However, cross-sectional data from this study show that although age and family history of OA were not associated with medial meniscal extrusion, our definition of past knee injury and weight (body weight, BMl and obesity) were significantly associated with prevalent medial meniscal extrusion. Obese individuals had nearly a fivefold increased risk for having meniscal extrusion than did nonobese individuals, and those satisfying our definition of past knee injury had a nearly fourfold increase in risk for having meniscal extrusion com-

Table 4

Associations between medial meniscal extrusion at baseline and change in cartilage volume over 2 years

\begin{tabular}{lll}
\hline Factor & Univariable $\beta(95 \% \mathrm{Cl})$ & Multivariablea $\beta(95 \% \mathrm{Cl})$ \\
\hline Medial tibial (\%) & $-2.29(-0.41 \text { to }-0.45)^{\star}$ & $-1.26(-2.95$ to +0.43$)$ \\
Medial femoral (\%) & $-0.73(-2.06$ to +0.59$)$ & $-0.87(-2.23$ to +0.49$)$ \\
Medial tibiofemoral (\%) & $-1.42(-2.66 \text { to }-0.17)^{\star}$ & $-1.18(-2.41$ to +0.05$)$ \\
Lateral tibial $(\%)$ & $-0.14(-1.69$ to +1.41$)$ & $+0.43(-1.09$ to +1.94$)$ \\
Lateral femoral $(\%)$ & $-0.28(-1.54$ to +0.98$)$ & $-0.34(-1.62$ to +0.94$)$ \\
Lateral tibiofemoral $(\%)$ & $-0.28(-1.45$ to +0.88$)$ & $-0.07(-1.22$ to +1.09$)$
\end{tabular}

The dependent variable was change in cartilage volume. aAdjusted for age, sex, offspring-control status, body mass index, past knee injury and baseline cartilage volume. bFurther adjusted for baseline tibial bone area and osteophytes. ${ }^{\star}$ Statistically significant associations. Cl, confidence interval. 
pared with those with no such history. These suggest that obesity and past knee injury are major risk factors for meniscal extrusion. It is possible that knee injury was in part caused by chronic knee instability resulting from an overuse of secondary stabilizers such as cruciate and collateral ligaments [19]. Moreover, women tended to be at greater risk for having medial meniscal extrusion than did men in multivariable analysis, although this did not reach statistical significance.

We have reported that tibial bone area and osteophytes are strongly associated with prevalent knee cartilage defects [17] and incident knee cartilage defects [15]. We found in this study that medial tibial bone area and osteophytes were also significantly associated with the prevalence of medial meniscal extrusion, suggesting that subchondral bone expansion may play an important role in the initiation not only of cartilage damage $[15,17]$ but also of meniscal extrusion. It is also possible that meniscal extrusion can induce subchondral bone expansion and osteophytes, because without the meniscus, which functions as an energy absorber, the increased contact between tibia and femur may contribute to remodelling of bone. However, we found that baseline medial meniscal extrusion was not associated with change in tibial bone area (Table 1 ), suggesting that tibial bone area is not affected during the early stages of meniscal extrusion.

With increasing meniscal extrusion, there may be increasing contact stress on the tibial and femoral articular cartilage, which theoretically might accelerate the development of cartilage damage. Berthiaume and coworkers [7] reported that OA patients with severe medial meniscal tear had greater loss of medial compartment cartilage volume than did those with no medial meniscal tear over 2 years. Raynauld and coworkers [9] further reported that severe meniscal extrusion was more prevalent $(73 \%)$ in OA patients who experienced more rapid loss of global knee cartilage volume, and severe medial meniscal extrusion was found to be an independent predictor of loss of medial compartment cartilage volume over 2 years. Hunter and coworkers [10] reported that meniscal malposition was significantly associated with increased risk for focal cartilage loss over 30 months in patients with symptomatic OA. Consistent with these findings in patients with disease, in the present study - conducted largely in individuals without OA - we found medial meniscal extrusion to be significantly associated with change in medial femoral and lateral tibial cartilage defects over 2 years after adjustment for age, sex, BMI, family history of $\mathrm{OA}$ and past knee injury. Furthermore, we found that medial meniscal extrusion was significantly associated with loss of medial tibiofemoral cartilage volume over 2 years after adjustment for the above factors as well as bone changes.

Our data suggest that tibial bone area and osteophytes may lead to meniscal extrusion, which in turn leads to joint space narrowing on radiography and knee cartilage defects and tibial cartilage loss. We found that meniscal extrusion was significantly associated with joint space narrowing in the unadjusted analysis and after adjustment for age, sex, OA family history and BMI. This is consistent with previous findings showing that meniscal extrusion (rather than meniscal compression) contributes to joint space narrowing $[2,3,9]$. However, the association between meniscal extrusion and joint space narrowing became nonsignificant after adjustment for tibial bone area and osteophytes. This suggests that subchondral bone changes leads to meniscal extrusion and joint space narrowing. Consistent with this, after adjustment for tibial bone area and osteophytes, the associations between meniscal extrusion and prevalent and incident defects in knee cartilage disappeared, and the association between meniscal extrusion and tibial cartilage loss decreased in magnitude. This suggests that meniscal extrusion is an intermediate variable on the pathway between bone change and cartilage damage.

The study has a number of potential limitations. It was primarily designed to examine genetic mechanisms of knee OA and utilized a matched design. The matching protocol was not adhered to in the present study, but adjustment for family history did not alter the results. Although the sample is a convenience sample, Miettinen [20] stated that for associations to be generalized to other populations, three key criteria including selection (inclusion/exclusion criteria for both offspring and controls are explicitly defined), sample size and adequate distribution of study factors need to be met, all of which are met by this study. Second, we cannot confirm the cross-sectional results in the longitudinal component of our study because of the low incidence rate; hence, studies of longer term will be required. Third, the amount of meniscal extrusion may be underestimated on MRI scans because of non-weight bearing knees and may have been more prevalent if the study had been carried out under load conditions. Pathological patterns such as joint laxity, axial deviation or varus-valgus deformity may also influence the measured values under non-weight-bearing conditions. Fourth, we asked all participants to wait for $15 \mathrm{~min}$ in the waiting room before MRI scan, but we did not record the intensity of load force and recovery time after running, walking, or sitting. This may influence the measurement of cartilage volume. However, a recent study [21] showed that these moderate activities had little impact on tibial and femoral cartilage deformation in vivo in healthy individuals. A fifth limitation is that our definition of past knee injury can be considered imprecise. However, we found in this study that it was significantly associated with meniscal extrusion, suggesting that the definition has predictive validity. Finally, measurement error may influence the results. However, scoring of meniscal extrusion, knee cartilage defects, volume, bone size and tibiofemoral radiographic score measurement was highly reproducible, suggesting this is unlikely.

\section{Conclusion}

This study suggests that increasing BMl and bone size, past knee injury, and osteophytes may be causally related to menis- 
cal extrusion. Most importantly, meniscal extrusion at baseline is associated with greater loss of knee cartilage over 2 years and this association is mostly mediated by subchondral bone changes, suggesting that extrusion represents one pathway between bone expansion and cartilage loss.

\section{Competing interests}

The authors declare that they have no competing interests.

\section{Authors' contributions}

$C D, G J, J M-P, J-P P, J-P R$ and FC participated in the design of the study. CD and FA carried out the measurement of cartilage volume, cartilage defects, bone size and/or meniscal extrusion. CD performed the statistical analysis and drafted the manuscript. All authors reviewed the manuscript, and read and approved the final manuscript.

\section{Acknowledgements}

We offer special thanks to the participants and orthopaedic surgeons who made this study possible. The role of Catrina Boon in coordinating the study is gratefully acknowledged. We should like to thank Martin Rush, who performed the MRI scans; Kevin Morris, for technical support; and Josée Thériault and André Pelletier, for meniscal reading. Support for the present study was provided by the National Health and Medical Research Council of Australia, Masonic Centenary Medical Research Foundation, and ArthroVision, Montreal, Canada.

\section{References}

1. Martel-Pelletier J, Lajeunesse D, Pelletier J-P: Etiopathogenesis of osteoarthritis. In Arthritis and Allied Conditions: A Textbook of Rheumatology 15th edition. Edited by: Koopman WJ. Baltimore, MA: Lippincott, Williams \& Wilkins; 2005:2199-2226.

2. Gale DR, Chaisson CE, Totterman SM, Schwartz RK, Gale ME Felson D: Meniscal subluxation: association with osteoarthritis and joint space narrowing. Osteoarthritis Cartilage 1999, 7:526-532.

3. Adams JG, McAlindon T, Dimasi M, Carey J, Eustace S: Contribution of meniscal extrusion and cartilage loss to joint space narrowing in osteoarthritis. Clin Radiol 1999, 54:502-506.

4. Miller TT, Staron RB, Feldman F, Cepel E: Meniscal position on routine MR imaging of the knee. Skeletal Radiol 1997, 26:424-427.

5. Lerer DB, Umans $\mathrm{HR}, \mathrm{Hu} \mathrm{MX}$, Jones $\mathrm{MH}$ : The role of meniscal root pathology and radial meniscal tear in medial meniscal extrusion. Skeletal Radiol 2004, 33:569-574.

6. Puig L, Monllau JC, Corrales M, Pelfort X, Melendo E, Caceres E: Factors affecting meniscal extrusion: correlation with MRI, clinical, and arthroscopic findings. Knee Surg Sports Traumatol Arthrosc 2006, 14:394-398.

7. Berthiaume MJ, Raynauld JP, Martel-Pelletier J, Labonte F, Beaudoin G, Bloch DA, Choquette D, Haraoui B, Altman RD, Hochberg $M$, et al:: Meniscal tear and extrusion are strongly associated with progression of symptomatic knee osteoarthritis as assessed by quantitative magnetic resonance imaging. Ann Rheum Dis 2005, 64:556-563.

8. Costa CR, Morrison WB, Carrino JA: Medial meniscus extrusion on knee MRI: is extent associated with severity of degeneration or type of tear? AJR Am J Roentgeno/ 2004, 183:17-23.

9. Raynauld JP, Martel-Pelletier J, Berthiaume MJ, Beaudoin G, Choquette D, Haraoui B, Tannenbaum H, Meyer JM, Beary JF, Cline $\mathrm{GA}$, et al:: Long term evaluation of disease progression through the quantitative magnetic resonance imaging of symptomatic knee osteoarthritis patients: correlation with clinical symptoms and radiographic changes. Arthritis Res Ther 2006, 8:R21.

10. Hunter DJ, Zhang YQ, Niu JB, Tu X, Amin S, Clancy M, Guermazi A, Grigorian M, Gale D, Felson DT: The association of meniscal pathologic changes with cartilage loss in symptomatic knee osteoarthritis. Arthritis Rheum 2006, 54:795-801.

11. Jones G, Ding C, Scott F, Cicuttini F: Genetic mechanisms of knee osteoarthritis: a population based case-control study. Ann Rheum Dis 2004, 63:1255-1259.

12. Jones G, Ding C, Scott F, Glisson M, Cicuttini F: Early radiographic osteoarthritis is associated with substantial changes in cartilage volume and tibial bone surface area in both males and females. Osteoarthritis Cartilage 2004, 12:169-174.

13. Jones $G$, Glisson M, Hynes K, Cicuttini F: Sex and site differences in cartilage development: a possible explanation for variations in knee osteoarthritis in later life. Arthritis Rheum 2000, 43:2543-2549.

14. Ding C, Cicuttini F, Scott F, Boon C, Jones G: Association of prevalent and incident knee cartilage defects with loss of tibial and patellar cartilage: a longitudinal study. Arthritis Rheum 2005, 52:3918-3927.

15. Ding C, Cicuttini F, Scott F, Cooley H, Boon C, Jones G: Natural history of knee cartilage defects and factors affecting change. Arch Intern Med 2006, 166:651-658.

16. Raynauld JP, Martel-Pelletier J, Berthiaume MJ, Labonte F, Beaudoin G, de Guise JA, Bloch DA, Choquette D, Haraoui B, Altman $\mathrm{RD}$, et al.: Quantitative magnetic resonance imaging evaluation of knee osteoarthritis progression over two years and correlation with clinical symptoms and radiologic changes. Arthritis Rheum 2004, 50:476-487.

17. Ding C, Garnero P, Cicuttini F, Scott F, Cooley H, Jones G: Knee cartilage defects: association with early radiographic osteoarthritis, decreased cartilage volume, increased joint surface area and type II collagen breakdown. Osteoarthritis Cartilage 2005, 13:198-205.

18. Breitenseher MJ, Trattnig S, Dobrocky I, Kukla C, Nehrer S, Steiner $\mathrm{E}$, Imhof $\mathrm{H}$ : MR imaging of meniscal subluxation in the knee. Acta Radiol 1997, 38:876-879.

19. Mohana-Borges AV, Resnick D, Chung CB: Magnetic resonance imaging of knee instability. Semin Musculoskelet Radiol 2005, 9:17-33.

20. Miettinen OS: Theoretical Epidemiology: Principles of Occurrence Research in Medicine Hoboken, NJ: John Wiley and Sons, Inc; 1985.

21. Eckstein F, Lemberger B, Gratzke C, Hudelmaier M, Glaser C, Englmeier $\mathrm{KH}$, Reiser $\mathrm{M}$ : In vivo cartilage deformation after different types of activity and its dependence on physical training status. Ann Rheum Dis 2005, 64:291-295. 